\title{
Periodontal Alteration of the Microcirculation and Hypercholesterolemia: A Possible Correlation?
}

\author{
Giuseppe Alessandro Scardina, DMD, Teresa Pisano, DMD, Antonino Cacioppo, DMD, \\ and Pietro Messina, MD
}

Objective: We evaluated the morphological and parametric characteristics of the periodontal microcirculation in patients diagnosed as having hypercholesterolemia and high levels of low-density lipoprotein (LDL).

Methods: Forty patients were recruited, 20 of whom were affected by hypercholesterolemia and 20 of whom were considered healthy. A videocapillaroscopic examination was carried out on the periodontal mucosa in the proximity of the frenulum (II, V sextant).

Results: The difference between the parameters of the hypercholesterolemia group and the control group was evaluated with the Mann-Whitney U-test for non-parametric ordinal data; the level of significance being $P<0.05$. The videocapillaroscopy documented extremely significant differences between the two groups, regarding the following parameters: total diameter of the loop $(P=0.0017)$, diameter of the afferent loops $(P=0.0004)$, diameter of the efferent loops $(P=0.00008)$ and periodontal density $(P=0.0001)$.

Conclusions: The capillaroscopic examination revealed a morphological alteration of the periodontal microcirculation in patients with hypercholesterolemia, which is an expression of peripheral vascular phlogosis.

Key Words: hypercholesterolemia, oral videocapillaroscopy, periodontal disease

$\mathrm{I}_{\mathrm{f}}^{\mathrm{n}}$ subjects with hypercholesterolemia, morphological and functional alterations are found in correspondence with the walls of arterial vessels, characterized by an inflam-

From the Department of Oral Sciences, University of Palermo, Palermo, Italy.

Reprint requests to Giuseppe Alessandro Scardina, DMD, Department of Oral Sciences, University of Palermo, “G. Messina” Via Del Vespro, 189 90127 Palermo, Italy. Email: scardina@odonto.unipa.it

The authors have no financial disclosures to declare and no conflicts of interest to report.

Accepted September 2, 2010.

Copyright (C) 2011 by The Southern Medical Association

0038-4348/0-2000/10400-0116 matory response. ${ }^{1}$ In particular, high levels of low-density lipoprotein (LDL) contribute to the microvascular dysfunction. $^{2}$

Recent studies have shown associations between periodontal disease and some systemic diseases. Systemic, local chronic, or acute infections can induce changes in the plasmatic concentration of cytokines, which in turn determines changes in the lipidic metabolism. Scientific evidence suggests that the local inflammatory processes provoke a systemic response in the host. ${ }^{3}$ Therefore, subjects with periodontal disease have an increased risk of developing metabolic syndrome. ${ }^{4}$ Some studies have shown an association between hypercholesterolemia and severe periodontal disease ${ }^{5,6}$ In addition, these patients display a dysmetabolic state characterized by a decreased level of highdensity lipoprotein (HDL) in the serum and an increase in the level of LDL. ${ }^{7}$ Recently, Fentoğlu has shown that subjects with light and moderate hyperlipidemia have higher parameter values for periodontal disease compared to normolipidemic subjects. ${ }^{8}$ According to Pohl et al, ${ }^{1}$ the degree of periodontal breakdown is positively correlated to the plasma cholesterol levels. Moeintaghavi et $\mathrm{al}^{9}$ found that hyperlipidemia might even be associated with periodontitis in healthy people.

\section{Key Points}

- Our findings showed an association between hyperlipidemia and periodontal morphological microcirculation.

- Local inflammatory processes may provoke a systemic response in the host, which means that subjects with periodontal disease may have an increased risk of developing metabolic syndrome.

- The observed changes in lipid metabolism could either be the cause or the consequence of periodontal disease. 
Table 1. Demographic characteristics of the patients enlisted in the study ${ }^{a}$

\begin{tabular}{lcc}
\hline & $\begin{array}{c}\text { Case } \\
\text { group }\end{array}$ & $\begin{array}{c}\text { Control } \\
\text { group }\end{array}$ \\
\hline $\mathrm{M} / \mathrm{F}$ & $6 / 14$ & $6 / 14$ \\
Age $(\mathrm{yr})$, mean $\pm \mathrm{SD}$ & $65.80 \pm 8.78$ & $60.33 \pm 13.61$ \\
Range & $48-77$ & $28-76$
\end{tabular}

${ }^{a} M / F$, male or female; $S D$, standard deviation.

The aim of this study is to evaluate the in vivo morphological characteristics of periodontal microcirculation through videocapillaroscopic examination in patients diagnosed with hypercholesterolemia and high-levels of LDL without periodontal disease, with the aim of revealing possible differences between them and healthy patients.

\section{Materials and Methods}

Forty subjects were examined in our laboratory (Table 1), 20 of whom were affected by hypercholesterolemia without pharmacological treatment (total cholesterol $>200 \mathrm{mg} / \mathrm{mL}$; LDL cholesterol $160<\mathrm{LDL}<190 \mathrm{mg} / \mathrm{mL}$ ) and 20 of whom were considered healthy. All subjects gave their consent in accordance with Italian law. The gingival index, as well as plaque index, was equal to zero for all patients participating in the study.

A blood test was done on hypercholesterolemic patients who had not had anything to eat or drink, which permitted us to measure their values of total and LDL cholesterol. When testing the level of LDL cholesterol, which is rarely measured directly, it can be calculated using the following formula: total (TOT) Cholesterol = HDL + LDL + VLDL (Very Low Density Lipoprotein), where VLDL is the quantity of cholesterol bound to VLDL lipoprotein. ${ }^{10}$ The VLDL cholesterol can be indirectly calculated with a very simple formula: VLDL $=$ Triglycerides $/ 5$. Putting together the two formulas,

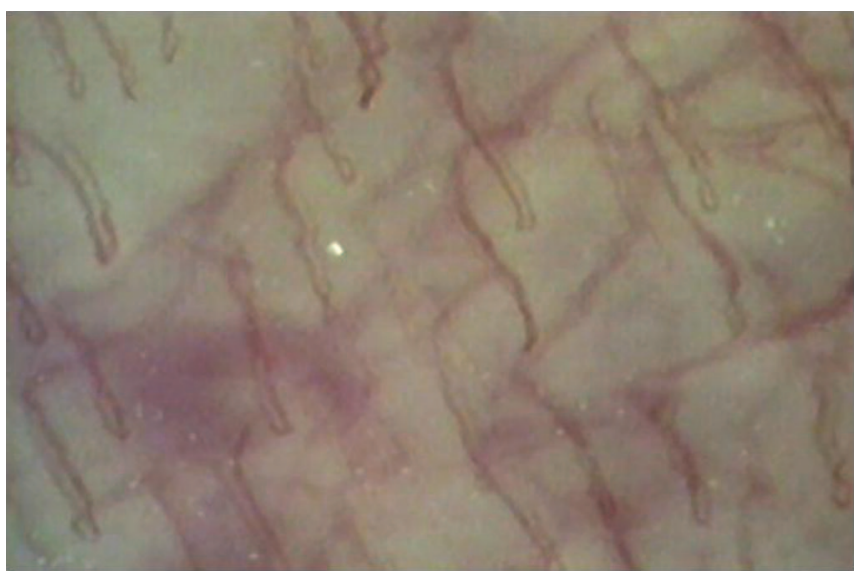

Fig. 1 Periodontal microcirculation in healthy subjects. Image $200 \times$.

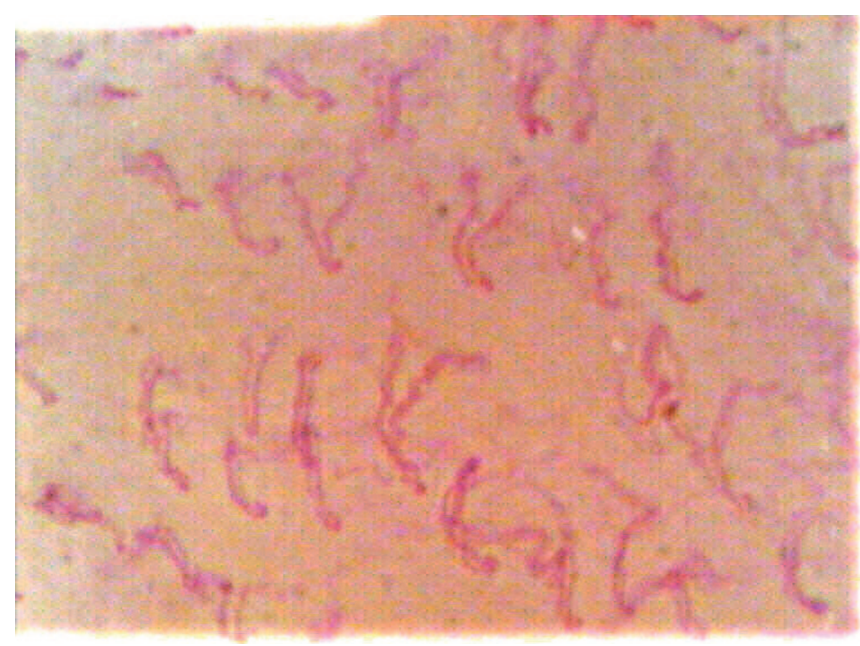

Fig. 2 Periodontal mucosa in patients affected by hypercholesterolemia. Image $200 \times$.

the following formula is obtained: TOT Cholesterol $=\mathrm{HDL}+$ LDL + (Triglycerides/5) from which the Friedewald formula can be easily drawn: LDL $=$ TOT Cholesterol $-(\mathrm{HDL}+$ Triglycerides/5).

The videocapillaroscopic examination was performed on all patients within a controlled environment which included light source, room temperature $\left(23^{\circ} \mathrm{C}\right)$, time of day (morning), sitting position, and operator. Each examination was repeated two times per patient for the area under investigation, regardless of whether the patient was in the control group or in the hypercholesterolemia group. This investigation area consisted of the periodontal mucosa in the proximity of the frenulum (II-V sextant).

Videocapillaroscopy consists of a central cold-halogen light source, usually a 100-watt lamp fitted with an automaticcontrol device to regulate luminosity, and a fibre-optic probe

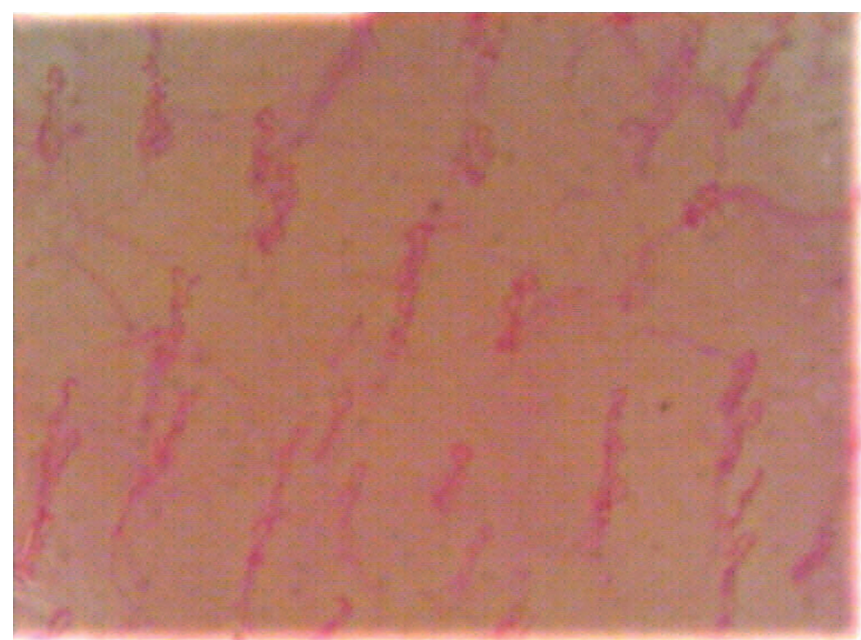

Fig. 3 Corkscrew capillary loops in periodontal mucosa of hypercholesterolemia patient. Image $200 \times$. 


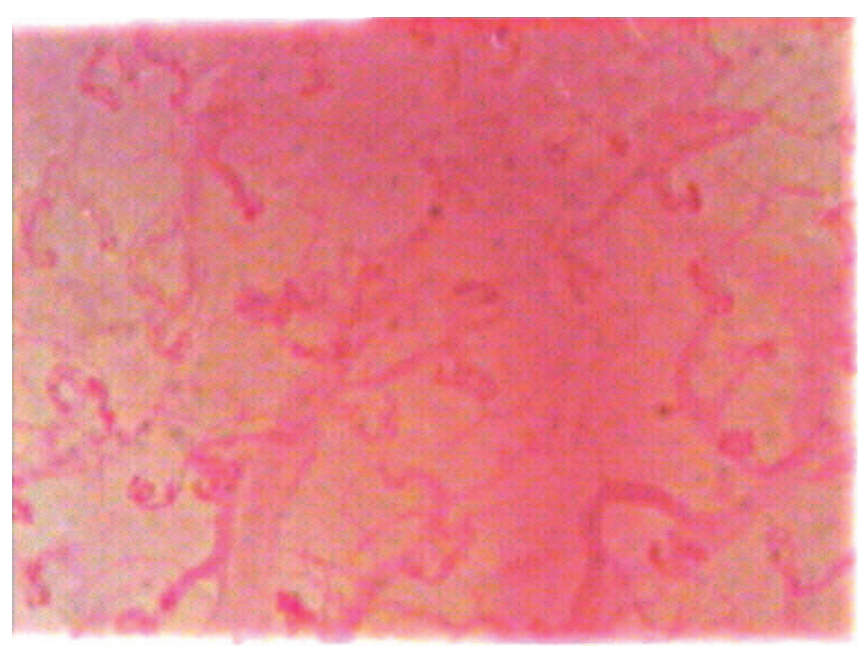

Fig. 4 Particularly tortuous capillary loops in periodontal mucosa of hypercholesterolemia patient. Image $200 \times$.

made up of a 2-meter flexible cable with a video-optic terminal; the terminal itself consists of a color microtelevision camera and a support holding different lenses. The lenses are made up of both contact and non-contact types with variable enlargements of $20 \times, 50 \times, 100 \times, 200 \times$, $500 \times$, and $1000 \times$. The image is then displayed on a highresolution color monitor.

The choice of lens was easy because studying the microcirculation of mucosa lends itself to using a contacttype lens. For the identification of the microangiotectonic, and for the definition of the type and group to which it belongs, a $200 \times$ optic was used. This allowed us to explore each point of the morphostructural characteristics of the capillaroscopic field.

Among the more important morphological parameters we analyzed by averaging the two observations per each ex-

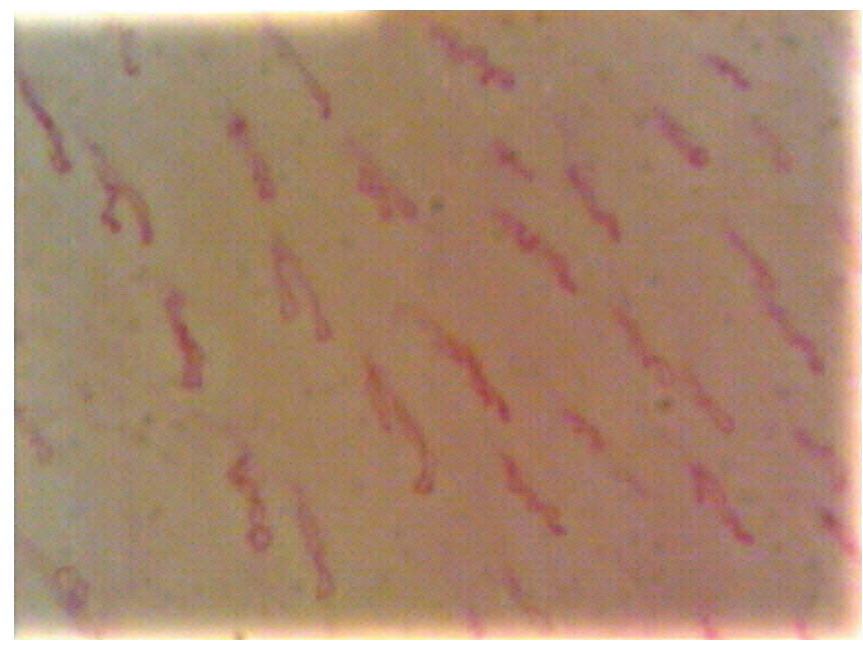

Fig. 5 Deer antler loops in periodontal mucosa of hypercholesterolemia patient. Image $200 \times$.

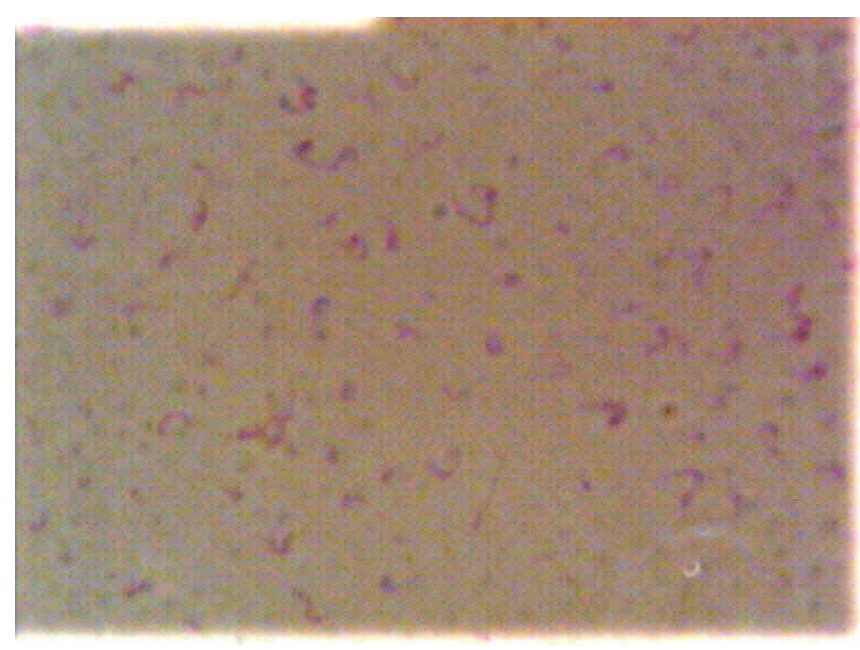

Fig. 6 Masticatory mucosa in patient affected by hypercholesterolemia. Image $200 \times$.

amined area were the length of the capillary loop, the diameter of the loop, the diameter of the afferent and efferent loops, the capillary density, and the morphology of the loops. ${ }^{11,12}$

\section{Results}

The architecture of the microcirculation of periodontal mucosa was found to be referable to type II group B of the Curri classification, with capillaries of slightly long loops arranged both parallel and perpendicular to the surface of the mucosa, all of uniform caliber, hairpin in shape, and winding back on themselves (Figs. 1 and 2). In addition, particularly tortuous (corkscrew) loops were also found (Fig. 3), as well as arborescent ones (bush-like, wound-up balls; Figs. 4-6). The periodontal microcirculation of some patients stood out for the presence of microhemorrhages,

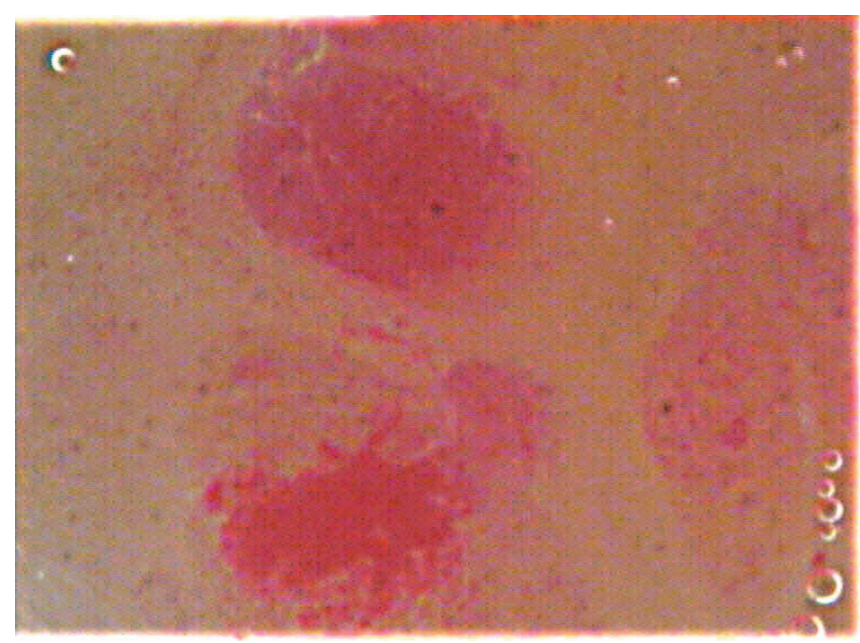

Fig. 7 Gingival mucosa and presence of microhemorrhages in patient affected by hypercholesterolemia. Image $200 \times$. 


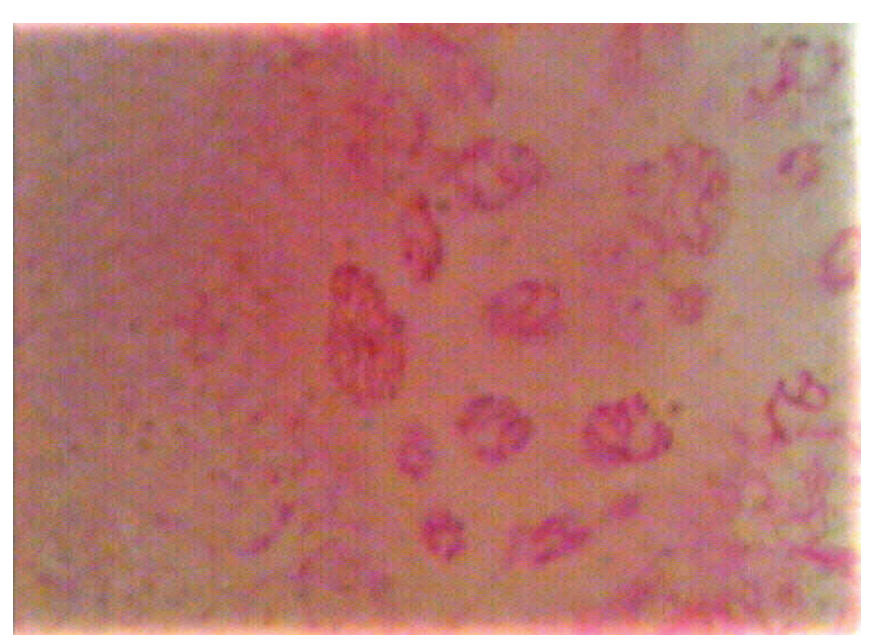

Fig. 8 Gingival mucosa and disorderly orientation of the capillary loops in patient affected by hypercholesterolemia. Image $200 \times$.

as a result of vascular disorder (Fig. 7), and for the alteration of normal architecture (Fig. 8).

In consideration of the morphofunctional alterations in the periodontal mucosa, including their gravity and frequency as evidenced by the capillaroscopic examination, an alteration of the capillaries can be seen. This architectural disorder can be observed by the slightly tortuous, arborescent capillary loops, and by the presence of sporadic microhemorrhages. A significant difference can also be seen in the diameter of the loops of the two groups examined.

The results of the statistical analysis of the data, divided by the parameters of interest, are shown in the following table (Table 2). The difference between the two groups, including parameters of the total diameter of the loop, the diameter of afferent loops, the diameter of efferent loops, and the periodontal density, was highly significant.

\section{Discussion}

Experimental and clinical data suggest that an increased level of LDL cholesterol is associated with damage to endothelial functioning. Hypercholesterolemia seems to convert the normal anti-inflammatory phenotype in microcirculation to a pro-inflammatory phenotype. In addition, it alters the endothelial function, decreasing the availability of nitric oxide, probably through the increase of oxygen and the production of free radicals. ${ }^{13}$ Research has shown a correlation between the immune system and hypercholesterolemia. The T-lymphocytes seem to be involved in a series of endothelial changes, which eventually leads to the accumulation of leucocytes in the postcapillary venule and to the endothelial dysfunction of the arterioles. ${ }^{14}$

Maglakelidze et al carried out a study on rabbits with hypercholesterolemia which revealed significant changes in the extracellular matrix and in the mucosa cells of the gums, as well as in the microcirculatory bed. Hypercholesterolemia damages the endotheliocytes, the subendothelial areas, and the basal membranes with changes in permeability. The contact of the lymphocytes and the plasmocytes with the vascular wall confirms the role that the triggers of the vascular factor play in damaging the vascular complex. ${ }^{15}$ Maklakelidze et al studied the morphological substratum of the gingival mucosa during the increase of the proatherogenic concentration of lipids, and the damage to the gums under the influence of an atherogenic diet. Using the planimetric method, the average rates of the number of capillaries, venules, and arterioles were defined, as well as the quantity of immunocompetent cells (macrophages and lymphocytes) in the subepithelial gingival connective tissue of rabbits with hypercholesterolemia. In the subepithelial layer of the gingival mucosa, a deformed lumen was observed. ${ }^{16}$

Acute infections are known to interfere with lipid metabolism, and the elevation of plasma triglycerides has been observed, especially in infections with Gram-negative bacteria. These changes are thought to be mediated by cytokines such as Interleukin-1 (IL1) or tumor-necrosis factors (TNF), which may be produced at the inflamed periodontal tissues in high quantities. These biological signaling molecules from local inflammation have a myriad of physiological effects, including promoting enhanced lipogenesis, increased lipolysis, and reduced lipid clearance. The end result is hyperlipidemia, or an accumulation of serum-free fatty acids (FFA), LDL, and thyroglobulin (TG).

A possible role for hyperlipidemia in the development of periodontitis is indicated in several studies. Hyperlipidemia is

Table 2. Overall results of the statistical analysis, carried out with the Mann-Whitney $U$ test $^{a}$

\begin{tabular}{|c|c|c|c|c|}
\hline $\begin{array}{l}\text { Parameter of } \\
\text { interest }\end{array}$ & $\begin{array}{c}\text { Cases } \\
(\text { median } \pm \text { SD) }\end{array}$ & $\begin{array}{c}\text { Control } \\
(\text { median } \pm \text { SD) }\end{array}$ & $P$ & Significance \\
\hline Length & $0.214 \pm 0.052$ & $0.196 \pm 0.064$ & 0.13 & NS \\
\hline Efferent diameter & $0.017 \pm 0.002$ & $0.010 \pm 0.003$ & 0.00008 & S \\
\hline Afferent diameter & $0.012 \pm 0.002$ & $0.007 \pm 0.002$ & 0.0004 & S \\
\hline
\end{tabular}

${ }^{a} S D$, standard deviation; $N S$, not significant; $S$, significant. 
known to cause hyperactivity of white blood cells. ${ }^{17}$ Hyperactivity of white blood cells, for example, because of an increased production of oxygen radicals, ${ }^{18}$ has been shown to be associated with progressive periodontitis in adults. ${ }^{19}$

We believe this to be the first scientific research within the odontostomatologic field that employs videocapillaroscopy for the morphofunctional evaluation of periodontal microcirculation in patients affected by hypercholesterolemia. Capillaroscopy has led to very important diagnostic results, in regard to the alterations of the periodontal-area microcirculation in patients with hypercholesterolemia, as opposed to healthy patients. In fact, capillaroscopic examination has allowed us to statistically determine a significant increase in the parameters of the diameters each of capillary loops, afferent loops, and efferent loops, and in the periodontal density of subjects with hypercholesterolemia at the $P<0.05$ level.

If considered all together, these results indicate the presence of a disturbance in periodontal microcirculation and an expression of phlogosis in the district. In patients with hypercholesterolemia, the endothelium is abnormal with increased levels of low-density lipoprotein. Microvascular alteration is a systematic process that manifests itself in a similar way within the vascular layers of multiple tissues within the organism. Our findings indicated an association between hyperlipidemia and periodontal morphological microcirculation. However, it is not clear yet whether the observed changes in lipid metabolism could be the cause or the consequence of periodontal disease. Further studies are needed to determine the exact role of hyperlipidemia and periodontal disease, and their relationship to each other.

\section{References}

1. Pohl A, Pohl C, Krause S, et al. Hyperlipidemia, atherosclerosis, and oral inflammatory disease. Acta Angiologica 1995;1:133-137.

2. Abularrage CJ, Sidawy AN, Aidinian G, et al. Evaluation of the microcirculation in vascular disease. J Vasc Surg 2005;42:574-581.

3. Beck JD, Offenbacher S, Williams R, et al. Periodontitis: a risk factor for coronary heart disease? Ann Periodontol 1998;3:127-141.

4. Banihashemrad SA, Moeintaghavi A, Rafighdoost A. Relationship between cholesterol and triglyceride blood values and periodontal parameters in patients of Mashhad health center. N Y State Dent J 2008;74:65-66.

5. Katz J, Chaushu G, Sharabi Y. On the association between hypercholesterolemia, cardiovascular disease and severe periodontal disease. $J$ Clin Periodontol 2001;28:865-868.

6. Nibali L, D'Aiuto F, Griffiths G, et al. Severe periodontitis is associated with systemic inflammation and a dysmetabolic status: a case-control study. J Clin Periodontol 2007;34:931-937.

7. Katz J, Flugelman MY, Goldberg A, et al. Association between periodontal pockets and elevated cholesterol and low density lipoprotein cholesterol levels. J Periodontol 2002;73:494-500.

8. Fentoğlu $\mathrm{O}, \mathrm{Oz}$ G, Taşdelen $\mathrm{P}$, et al. Periodontal status in subjects with hyperlipidemia. J Periodontol 2009;80:267-273.

9. Moeintaghavi A, Haerian-Ardakani A, Talebi-Ardakani M, et al. Hyperlipidemia in patients with periodontitis. J Contemp Dent Pract 2005; $6: 78-85$.

10. Nauck M, Warnick GR, Rifai N. Methods for measurement of LDLcholesterol: a critical assessment of direct measurement by homogeneous assays versus calculation. Clin Chem 2002;48:236-254.

11. Scardina GA, Carini F, Messina P. Oral capillaroscopy: a new diagnostic method. Reumatismo 2005;57:295-304.

12. Scardina GA, Fucà G, Messina P. Microvascular characteristics of the human interdental papilla. Anat Histol Embryol 2007;36:266-268.

13. Stokes KY, Cooper D, Tailor A, et al. Hypercholesterolemia promotes inflammation and microvascular dysfunction: role of nitric oxide and superoxide. Free Radic Biol Med 2002;33:1026-1036.

14. Stokes KY. Microvascular responses to hypercholesterolemia: the interactions between innate and adaptive immune responses. Antioxid Redox Signal 2006;8:1141-1151.

15. Maglakelidze N, Galogre A, Tsagareli Z. Functional-morphologic aspects of changes of mucosal gingiva microcirculatory bed vessels in experimental gingivitis against the background of hypercholesterolemia. Georgian Med News 2005;121:71-74.

16. Maklakelidze NN, Gogiashvili LE, Gorgoshidze GG, et al. Morphometric characteristic of the subepithelial connective tissue of the rabbit gingiva under the experimental gingivitis on the background of hypercholesterinemia. Georgian Med News 2007;145:81-85.

17. Krause S, Pohl A, Pohl C, et al. Increased generation of reactive oxygen species in mononuclear blood cells from hypercholesterolaemic patients. Thromb Res 1993;71:237-240.

18. Gustafsson A, Asman B. Increased release of free oxygen radicals from peripheral neutrophils in adult periodontitis after Fc delta-receptor stimulation. J Clin Periodontol 1996;23:38-44.

19. Cutler CW, Shinedling EA, Nunn M, et al. Association between periodontitis and hyperlipidemia: cause or effect? J Periodontol 1999;70: $1429-1434$ 